\title{
Summarized Review on Dermatomyositis: Demonstrating Modes of Diagnosis \& Current Therapeutic Managements
}

\author{
R. Ghosh ${ }^{1}$, D. Ghosh ${ }^{2}$ \\ ${ }^{1}$ MBBS (FINAL), GSL Medical College and General Hospital, Rajahmundry, Andhra Pradesh \\ ${ }^{2} \mathrm{MD}$ (AY), Ph.D. (C.U), Superintendent and Ex-officio professor, Viswanath Ayurved Mahavidyalaya \& \\ Hospital, Kolkata \\ Corresponding Author: R. Ghosh
}

\begin{abstract}
Dermatomyositis (DM) is an autoimmune idiopathic inflammatory myopathy characterized by distinctive skin lesions, specific autoantibodies, and, most importantly, subacute symmetrical proximal muscle weakness and inflammation. Clinically amyopathic DM is a type of DM that presents with only characteristic skin lesions and no muscle disease. Heliotrope rash, erythematous rash over the face, erythematous scaly papules over the interphalangeal and metacarpophalangeal joints (Gottron's papules), erythema over the same joints (Gottron's sign), photosensitive pruritic erythema over the anterior chest and neck (Vsign), erythema extending to the shoulders and malignancy, respiratory disease, and cardiac disease are all causes of mortality and morbidity in dermatomyositis. Depending on the patient's parameters, management may include photoprotection and medications such as systemic corticosteroids, antimalarials, mycophenolate mofetil, biologicals, and IV immunoglobulins.
\end{abstract}

Keyword: dermatomyositis, Gottron's papules, heliotrope rash, inflammatory myopathy

\section{INTRODUCTION}

Dermatomyositis is an idiopathic inflammatory myopathy characterized by a unique pattern of skin involvement. Women suffer from DM 2 to 3 times as frequently as men. The disease begins with a bimodal distribution of 5 to 14-year-olds and 45 to 60-year-olds. Despite this, adult-onset DM is considered as a paraneoplastic syndrome because this condition may signal the presence of cancer. The DM condition is progressive, chronic, and autoimmune. Patients have a skin rash and symmetric proximal muscle inflammation and weakness. A cutaneous symptom will usually occur before or along with muscle inflammation. ${ }^{(9)}$ Changes in the skin can occur before muscle symptoms appear. About $21 \%$ of the time, there is little to no muscle involvement. DM is a connective tissue disease, like other connective tissue diseases such as rheumatoid arthritis, eosinophilic fasciitis, Sjogren's syndrome, scleroderma, and lupus erythematosus. (10)(7)(8) However, in DM, the skin rash is typically the first presenting symptom. DM can take a variety of forms, from minor constitutional symptoms like fever and malaise to life-threatening cancers. DM patients are estimated to have a 6-fold increase in cancer risk following the first year of diagnosis. Cancer only occurs in adult-onset patients who display skin manifestations. Diagnosis is most dangerous early on. Non-Hodgkin lymphoma is the most prevalent of the various types of malignancies. Approximately $60 \%$ of patients will exhibit symptoms of DM before malignant neoplasm development. Up to $50 \%$ of patients will develop interstitial lung disease. Thoracic muscle 
weakness may also cause dyspnea in some patients, whereas skeletal muscle weakness causes dysphagia. ${ }^{(1)}$

$\mathrm{DM}$ is a distinct entity that is distinguished by a characteristic rash that occurs in conjunction with, or more often before, muscle weakness. Symptoms include a blue-purple discoloration on the upper eyelids with edema (heliotrope rash), a flat red rash on the face and upper trunk, and erythema of the knuckles with a raised violaceous scaly eruption (Gottron's sign) among other things. As well as on other body surfaces, the erythematous rash can appear on the knees, elbows, malleoli, neck, and anterior chest (often in a V sign), or the back and shoulders (shawl sign). The rash may worsen after being exposed to the sun for an extended period. In some patients, the rash is itchy and pruritic, particularly on the scalp, chest, and lower back. In addition, dilated capillary loops at the base of the fingernails are a distinguishing feature. ${ }^{(11)}$ Symptoms include irregular, thickened, and distorted cuticles, and the lateral and palmar areas of the fingers may become rough and cracked, with irregular, "dirty" horizontal lines, resembling those of a mechanic. (13) Strength can be mild, moderate, or severe enough to cause quadriparesis, depending on the severity of the weakness. It is common to find significant perivascular and perimysial inflammation in muscle biopsy samples taken from patients with this type of condition. DM is usually found on its own, but it can occur in conjunction with scleroderma and mixed connective tissue disease. Patients suffering from eosinophilia-myalgia syndrome, which is caused by the ingestion of contaminated 1tryptophan, have developed fasciitis and thickening of the skin, which are similar to the symptoms seen in chronic cases of dermatomyositis. $^{(1)(2)}$

\section{Pathophysiology}

The cause of dermatomyositis is largely unknown. The complement system, like many autoimmune diseases, appears to be the driving force behind endothelial and muscular damage caused by the interaction of genetic susceptibility and the environment. Polymorphisms in the tumor necrosis factor promoter and an allele of the HLADQA gene have been identified as risk factors in genetic studies. Whether these genes or others are to blame, environmental stressors trigger changes that set the stage for the disease once a genetic foundation has been established. ${ }^{(11)}$ Drugs (hydroxyurea, phenytoin, nonsteroidal antiinflammatory drugs, and statins), viruses (human immunodeficiency virus, Escherichia coli, and Coxsackie virus), malignancy, and ultraviolet radiation are some of the known environmental triggers. Autoreactivity (reaction against our cells) begins in response to genetic and environmental cues. Complement pathways generate enzymes, activate immune complexes, and eventually generate membrane attack complexes. The most important component for such a powerful system is regulation because the complement system can lyse cells, clear debris, activate cytokines, and initiate inflammatory mediators. ${ }^{(18)}$ Autoantibody proliferation is caused by complement deficiency, whereas tissue damage is caused by complement overactivation. (12) When complement defenses fail, as they do in DM, the body's cells, which aren't normally antigenic, start attacking. The formation of autoantibodies begins. Complement system components and membrane attack complex cells can be found in patients early in the disease process, as expected. These components cause cell death in endothelial cells. ${ }^{(14)}$ Interface dermatitis is a condition that begins at the dermo-epidermal junction and manifests itself in histologic samples. Basal cell vacuolization (spaces and cavities between basal cells and dermis), apoptotic keratinocytes (dead keratinocytes and their waste), and inflammatory infiltrate are all common findings. 10 Cytokines, such as type 1 and 2 interferons, are produced in excess. Interferons have been linked to a variety of autoimmune diseases and chronic inflammation, and they have the potential to 
harm endothelial cells as well as myofibersin DM. The complement system is also linked to inflammation, which damages capillaries and results in ischemic muscle damage. Autoantibodies specific to myositis have been found in 50 percent to 70 percent of DM patients. Muscle enzyme levels in DM patients are frequently abnormal, resulting in muscle weakness, disability, and contractures. More research is needed to figure out exactly how these disease markers interact to cause DM. Sixty percent of patients seek treatment when both muscle and skin symptoms become problematic, while thirty percent of patients only have a skin rash. This means that $90 \%$ of DM patients will have the typical cutaneous features when they first present. Even when muscle weakness is suppressed, the DM rash causes severe pruritus and burning, which can be refractory to treatment. According to a recent study, DM patients' quality of life, energy level, depression, and mood scores were significantly lower than those of patients with similar inflammatory skin diseases and chronic health conditions such as hypertension, recent myocardial infarction, or diabetes. DM Patients who complain of muscle weakness and say it's getting harder to get out of a chair, comb their hair, or climb the stairs etc. ${ }^{(1)}$

\section{Immunopathogenesis of DM:}

Activation of complement, possibly by autoantibodies, against endothelial cells and formation of $\mathrm{C} 3$ via the classic or alternative pathway.

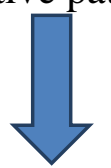

In response to the activation of $\mathrm{C} 3$, membrane attack complexes (MAC) are formed, which are then deposited in and around the endothelial cell wall of the endomysial capillary wall. ${ }^{(15)}$

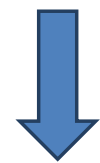

Deposition of MAC leads to the destruction of capillaries, ischemia, or microinfarcts, most prominent in the periphery of the fascicles, and perifascicular atrophy.

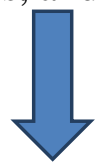

B cells, plasmacytoid dendritic cells, CD4 T cells, and macrophages are all involved in the movement of cells from the circulatory system to the muscular system. Cytokines released by mononuclear cells stimulate the expression of vascular cell adhesion molecule (VCAM) and intercellular adhesion molecule (ICAM) on the endothelium of the blood vessels. ${ }^{(15)}$

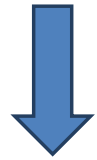

It is believed that integrins, specifically very late activation antigen (VLA)-4 and lymphocyte function-associated antigen (LFA)-1, bind VCAM and ICAM, allowing T cells and macrophages to infiltrate muscle through the endothelial cell wall. ${ }^{(6)(15)}$

\section{Clinical features:}

The following are the six most prominent skin manifestations of DM3:

1. Gottron's papules are raised, smooth, indurated, reddish violet lesions that appear primarily on bony prominences such as the knuckles, elbows, and knees. Gottron's papules are a type of keratosis. Also, they may appear on the sides of fingers or the tips of fingers. As 
a point of reference for differential diagnosis, Gottron's papules on the knuckles are present in 60 percent to 80 percent of dermatomyositis patients; on the other hand, cutaneous lesions on the knuckles are uncommon in patients with lupus. This symptom is pathognomonic for dermatomyositis. ${ }^{(10)}$

2. The heliotrope rash is a macular, reddish-purple rash that appears on the eyelids and is named after the small purple flower Heliotrope peruvianum, which grows in Peru. Periorbital edema is a symptom of this condition. Up to $60 \%$ of patients will develop a heliotrope rash, which can manifest as anything from dilated eyelid veins to a violet, edematous rash in some cases. It is also possible to experience scaling. This symptom is also pathognomonic for dermatomyositis. ${ }^{(1)(4)(7)}$

3. Violaceous/erythematous pruritic macular rash can affect any part of the body, but it is more common in sunexposed areas of the body. In addition to the bony prominences of the knuckles and elbows, the face, neck (V-sign), back (Shawl sign), lateral hips (holster sign), and extensor arms can all be affected. ${ }^{(8)}$ The macules can be found in a diffuse, confluent, or patchy pattern; they are usually symmetrical, and they are associated with severe and sometimes debilitating pruritus and itching. It is possible that scaling will occur. The initial assault may result in hyperkeratosis, pigment changes, and ulcerations, which may manifest themselves over time. As a point of distinction in the differential diagnosis, lupus erythematosus does not manifest itself with pruritus. ${ }^{(4)}$

4. Periungual telangiectasias are visible blood vessels that appear as irregular splinters on the proximal nail folds, and they are frequently associated with ragged, dystrophic cuticles. ${ }^{(10)}$

5. Scalp alopecia with scaly, reddish violet lesions is a condition that can occur.
6. Poikiloderma (from the Greek words poikilo, meaning irregular, and derma, skin), is a condition in which the skin becomes finely variegated with areas of hyper- and hypopigmentation, as well as areas of telangiectasia and atrophy. Typically, this manifests itself in the same sun-exposed areas where the violaceous/erythematous rash manifested itself in late-stage disease. (1)(4)(9)

\section{Other associated manifestations:}

Manifestations that are not restricted to the muscles and skins: These are some of the symptoms that may be present to varying degrees in patients with dermatomyositis:

1. The presence of systemic symptoms such as fever, malaise, weight loss, arthralgia, and Raynaud's phenomenon, particularly when inflammatory myopathy is associated with a connective tissue disorder, is of particular concern. ${ }^{(22)(11)}$

2. Joint contractures, which are most common in dermatomyositis and especially in children.

3. The involvement of the oropharyngeal muscles and the upper esophagus, particularly in dermatomyositis, results in dysphagia and gastrointestinal symptoms ${ }^{(19)}$.

4. Atrioventricular conduction defects (AVCDs), tachyarrhythmias (fast heartbeats), dilated cardiomyopathy (abnormally small left ventricular ejection fraction), and congestive heart failure, which may occur infrequently as a result of the disease itself or as a result of hypertension associated with longterm glucocorticoid treatment ${ }^{(18)(11)}$

5. Pneumonitis (e.g., from methotrexate) is a type of lung infection caused by a lack of strength in the thoracic muscles, interstitial lung disease, or a druginduced pneumonitis. It can cause dyspnea, nonproductive cough, and aspiration pneumonia in patients. As described below, interstitial lung disease 
(ILD) can occur before or early in the course of myopathy and can affect up to $10 \%$ of patients with PM or DM. The majority of these patients have antibodies to t-RNA synthetases, which can cause the disease to progress. ${ }^{(19)}$

6. Subcutaneous calcifications in dermatomyositis patients, which can occasionally extrude onto the skin and cause ulcerations and infections. (22)(11)

7. Arthralgias, synovitis, or deforming arthropathy with a subluxation in the interphalangeal joints, which can occur in some patients with dermatomyositis and Parkinson's disease who also have Jo-1 antibodies, can occur. ${ }^{(4)}$

\section{Differential diagnosis:}

Numerous differential diagnoses can be made based on the clinical presentation of DM. Because both are classified as idiopathic inflammatory myopathies and both are characterized by muscle abnormalities, DM and polymyositis (PM) are frequently confused when a patient presents with DM. DM, on the other hand, is distinguished from PM by cutaneous involvement. Furthermore, DM is more frequently linked to an underlying malignancy than PM, with reported rates ranging from $15 \%$ to $23 \%$. According to one study, 9 percent of people with PM were diagnosed with one of 42 cancers at the same time or shortly after they were diagnosed with PM. Cancer was the leading cause of death in 14 percent of the PM cases in the study. In the same study, 15 percent of the DM population was diagnosed with cancer, but cancer was the leading cause of death in 40 percent of the cases. To successfully diagnose DM, many cutaneous diseases must be ruled out in addition to PM as a differential diagnosis. Systemic lupus erythematosus, dermatitis, tinea corporis, psoriasis, and rosacea are just a few examples. Apart from diagnostic testing, distinguishes itself from many of these skin disorders by its pathognomonic heliotrope rash and involvement of proximal muscle weakness. ${ }^{(6)}$

\section{Diagnosis:}

\section{Blood investigation:}

In suspected cases of dermatomyositis, a complete blood count with an erythrocyte sedimentation rate (ESR), routine urine tests, a full biochemical profile that includes the presence of rheumatoid factor or inflammation markers, a chest X-ray, and fecal occult blood should be performed.

A raised ESR is present in half of the patients, and one-fifth of the patients have rheumatoid factor positivity, which is particularly prevalent in patients with overlap syndrome.

The level of creatine excretion in the urine can be measured to determine the severity of myositis, though it is elevated in steroid myopathies. Elevated serum myoglobin levels are also observed in patients with dermatomyositis. ${ }^{(6)}$

\section{Muscle enzymes:}

Creatine kinase $(\mathrm{CK})$ is the most sensitive enzyme and can be elevated by up to 50 times when the muscle is stressed. Even if there is no physical damage to the muscle, it is highly specific for DM in these circumstances. Increased levels of lactate dehydrogenase, aspartate transaminase, alanine transaminase, and aldolase, among other enzymes, have been reported. In most cases, muscle strength improves while muscle enzyme levels decrease, but this is not always the case. Because of this, improvements in muscle strength should be used to determine response to therapy rather than reductions in creatine kinase levels (CK). ${ }^{(1)(6)(5)}$

\section{Electromyography (EMG):}

Electromyography (EMG) is not particularly useful in confirming the diagnosis of dermatomyositis. An active muscle involvement site can be identified by a myopathic pattern with multiple, shortduration, small-amplitude motor unit action potentials if they are present, which can then be used to guide the selection of a suitable muscle biopsy site. ${ }^{(5)}$ 


\section{Muscle biopsy:}

An open muscle biopsy is taken from the site of the most severe muscle weakness or tenderness, or the site of the most significant EMG changes.Atrophic perifascicular atrophy is caused by necrosis and phagocytosis of the muscle fibers, which are visible as microinfarcts. ${ }^{(5)(1)}$

\section{Magnetic resonance imaging:}

MRI of the proximal muscles may reveal edema, which can be used to assess the extent of muscle disease as well as to guide the location of the muscle biopsy site on MRI. ${ }^{(1)}$

\section{Skin biopsy:}

A skin biopsy is usually performed in the case of amyopathic dermatomyositis, and it reveals a perivascular inflammatory infiltrate with lymphocytic infiltration at the dermo-epidermal junction, which is indicative of the disease. It is difficult to tell the difference between this and SLE. ${ }^{(2)}$

\section{Autoantibodies:}

Antinuclear antibodies (ANAs) are present in approximately $80 \%$ of dermatomyositis patients, but they are not associated with any significant diagnostic or prognostic value. Many antibodies found in DM are cytoplasmic, which means they will not result in a positive ANA. Myositisspecific antibodies, which aid in the definition of the phenotype of DM, are divided into three groups: anti-synthetase antibodies, which are the most common, followed by anti-signal recognition particle (SRP) antibodies, and anti-Mi2 antibodies. Anti-synthetase antibodies are the most common, followed by anti-signal recognition particle (SRP) antibodies, which are the second most common. ${ }^{(19)(15)}$

The anti-Jo1 antibody is the most common anti-synthetase antibody, with the others including anti-PL12, anti-PL7, anti$\mathrm{EJ}$, anti-OJ, anti-HA, anti-KS, and anti-ZO. The presence of these enzymes is referred to as anti-synthetase syndrome, and it is characterized by symptoms such as
Raynaud's syndrome, interstitial lung disease, mechanic's hands, fever, and arthritis. ${ }^{(20)(15)}$ Those who have anti-SRP antibodies have severe, acute resistant dermatomyositis with cardiac involvement, acute necrotizing myopathy, and rare cutaneous manifestations, among other things. (21)(15) It is highly specific for dermatomyositis to have anti-Mi2 antibody positivity, and these patients have classic cutaneous features, significant cuticular changes, a lower risk of myopathy, and a favorable response to treatment. (22) Antibodies associated with myositis, such as anti-PM / Scl , anti-Ro, anti-U1RNP, anti$\mathrm{Ku}$, and anti-La, may aid in the detection of an overlapping connective tissue disorder in patients with myositis. ${ }^{(2)}(15)$

\section{Tests to Rule Out Involvement of Other Organs and Malignancy}

To investigate ILD, which is a leading cause of death in dermatomyositis, pulmonary function tests and highresolution computed tomography are required. On the PFT, a restrictive pattern can be seen, which is also seen in respiratory muscle weakness. In ILD and malignancy, bronchoscopy and bronchoalveolar lavage can be used to rule out drug hypersensitivity, infection, and nonresponse to treatment. A lung biopsy can be used to rule out malignancy or infection, as well as to assess how well ILD is responding to treatment. A baseline ECG can be used to check for heart problems like conduction defects, ST-T changes, and arrhythmias. An echocardiogram and cardiac magnetic resonance imaging (MRI) can be used to locate the sites of myocardial inflammation. A barium swallow, esophageal manometry, and esophagogastroscopy can all be used to determine esophageal involvement. Nailfold capillaroscopy can be used to assess treatment response. Increased severity of skin disease, diaphragmatic weakness, dysphagia, and the absence of extra muscular systemic features such as ILD all increase the risk of malignancy. In all adult 
patients, computed tomography scans of the abdomen, pelvis, thorax, and neck may be required. A positron-emission tomography (PET) scan can be done if there is a high suspicion of malignancy. Calcinosis is another complication that is more common in juvenile DM patients who have had the disease for a longer time. It can be detected with simple radiography. ${ }^{(2)}$

Bohan and Peter criteria for the diagnosis of dermatomyositis

1. Symmetrical proximal muscle weakness

2. Muscle biopsy evidence of myositis

3. Elevation in serum skeletal muscle enzymes

4. Characteristic electromyography pattern of myositis

5. Typical rash of dermatomyositis

Definite: 5 plus any three of 1-4, Probable: 5 plus any two of 1-4, Possible: 5 plus any one of 1-4. ${ }^{(2)}$

\section{Treatment:}

Dermatomyositis is almost completely treatable, especially in children, though some patients have long-term or lifelong residual symptoms, necessitating long-term immunosuppression. Early aggressive treatment can help to reduce the occurrence of myopathy and calcinosis. The use of sunscreens and protective clothing, as well as avoiding peak-hour sun exposure, can help to prevent the exacerbation of skin lesions in DM. In the early stages of the disease, bed rest is advised. Physical therapy is required for rehabilitation after the active phase of the disease has passed. ${ }^{(17)}$

Antimalarial agents, topical steroids, and antihistamines are the first-line therapies in patients with cutaneous DM sun protection.

Oral corticosteroids, which can be tapered, and other immunosuppressive agents like methotrexate, mycophenolate mofetil, and azathioprine are examples of second-line therapy. Intravenous immunoglobulins can be given to patients who do not respond to these medications (IVIg). Rituximab is the next treatment option. Oral tacrolimus and sirolimus have been shown to improve myopathy symptoms. ${ }^{(17)}$

Also used were thalidomide, dapsone, and oral calcineurin inhibitors. In patients with refractory CADM, therapies like plasmapheresis, total-body irradiation, and stem cell transplantation have been used. $^{(2)(1)}$

\section{Antimalarial Medications}

Patients with primarily skin involvement can begin hydroxychloroquine (HCQ) treatment at a dose of $6.5 \mathrm{mg} / \mathrm{kg} / \mathrm{day}$ or less for two months. In CADM, HCQ can be combined with mepacrine with good results. Quinacrine, at a dose of $100 \mathrm{mg}$ per day, can be used if it is ineffective. Chloroquine at a dose of $3.5 \mathrm{mg} / \mathrm{kg} /$ day or less can be used in patients who had a reaction or were not responsive to HCQ. (2)(19)

\section{Corticosteroid:}

When there are both cutaneous and muscle involvement, only muscle involvement, extensive skin disease, or the patient has not responded to antimalarial agents for more than 2 months, systemic corticosteroids are the first line of treatment. If the muscle disease is life-threatening or oral prednisolone is ineffective, pulse therapy with IV methylprednisolone at $0.5-$ $1 \mathrm{~g} /$ day for three days can be followed by oral prednisolone at $0.5-1 \mathrm{mg} / \mathrm{kg} / \mathrm{day}$. Even the most potent topical steroids haven't shown many benefits in CADM, but topical tacrolimus ( 0.1 percent) has. Within 1 or 2 weeks of starting systemic corticosteroids, the muscle rash and weakness begin to improve. Systemic steroids must be given for a long time because DM is a chronic disease. The goal of treatment is to taper the dose and eventually stop steroids due to the side effects of long-term steroid therapy. Patients are then switched to different immunosuppressive medications. (2)(18)

\section{Antipruritic:}

Patients with DM may experience severe pruritus, which can be treated with 
topical agents like camphor, pramoxine, lidocaine, and menthol, as well as oral antihistamines and amitriptyline.

Agents that suppress the immune system in other ways Oral steroids are gradually tapered after 3 weeks to a month of treatment and other immunomodulators are added to prevent steroid-related toxicity. (3)(2)(22)

\section{Methotrexate:}

Methotrexate is the first-line steroidsparing agent in the treatment of dermatomyositis. It's taken orally or subcutaneously in doses ranging from 7.5 to $25 \mathrm{mg}$ per week. It is not useful for rapid control of acute or progressive DM because it takes 6-8 weeks to show effect. It's important to keep an eye on hepatic and pulmonary parameters. Azathioprine is given at a dose of $2-3 \mathrm{mg} / \mathrm{kg} / \mathrm{day}$ and has been shown to reduce the dose of steroids in long-term follow-up data. In refractory cases, cyclosporine at a dose of 3-5 $\mathrm{mg} / \mathrm{kg} /$ day has been used in combination with IVIg for relapsed or resistant disease. Hypertension, infection, and renal toxicity are all common side effects. ${ }^{(21)(22)}$

In patients who are resistant or intolerant to other immunosuppressants, mycophenolate mofetil at a dose of 500$1500 \mathrm{mg} /$ day is effective, and it has also been used in combination with IVIg in severe myositis cases. Hepatic and hematological toxicities should be monitored. ${ }^{(2)(20)(21)}$

\section{Intravenous immunoglobulin:}

If antimalarial drugs and other immunosuppressants do not work, intravenous immunoglobulin (IVIG) at a dose of $2 \mathrm{~g} / \mathrm{kg}$ given for 2-5 days every month for 3 months can be given. It helps patients with refractory myositis, especially those who have dysphagia. The cost of this treatment is a limiting factor. ${ }^{(2)(15)}$

\section{Rituximab}

Rituximab, given twice a week for two weeks, is effective in patients with recalcitrant or severe disease. ${ }^{(6)(2)(20)}$

\section{Miscellaneous}

Dapsone's anti-inflammatory properties may be beneficial in the treatment of resistant cutaneous DM. Antiestrogens like tamoxifen and anastrozole can help with skin lesions, but there is a risk of relapse if you stop taking them. The poikilodermatous erythema in DM can be reduced using a pulsed dye laser. Efalizumab, a monoclonal antibody against CD11a, is effective in reducing erythema around exposed areas. Leflunomide (20 $\mathrm{mg}$ /day) is effective in the treatment of recalcitrant DM skin lesions. The same medications are used to treat juvenile dermatomyositis as they are for adults. The mainstay of treatment is a combination of corticosteroids and weekly methotrexate. (2)(18)(19)

Diet

Muscle function has been shown to improve significantly when creatine supplements are combined with exercise. Renal toxicity should be considered, and it should not be given to anyone with a serious underlying medical condition or kidney disease. ${ }^{(2)(17)}$

\section{Exercise}

Even those with myositis who are responding to treatment have a chance of becoming disabled. All myositis patients must follow an exercise regimen. Exercises should be avoided during the acute phase except to maintain range of motion and prevent pressure sores and hypostatic pneumonia. Exercise is required for muscle function and endurance in a stable patient. It also aids in the movement toward oxidative metabolism, which reduces muscle inflammation and fibrosis by lowering the expression of proinflammatory and profibrotic factors. ${ }^{(16)}$ 
For DM, a multifaceted treatment strategy is required, depending on the extent of skin, muscle, and lung involvement, as well as other organ involvement, comorbidities, and medication side effects. For the treatment of DM, dermatologists and rheumatologists must collaborate. Specialists in respiratory medicine and other fields may be needed. Immunosuppressants like methotrexate, cyclosporine, and azathioprine are used for long-term therapy to reduce the side effects of steroids, and IVIg is useful for acute DM. ${ }^{(2)(19)}$

\section{CONCLUSION}

Dermatomyositis (DM), an idiopathic inflammatory myopathy, is characterized by skin manifestation. Both juvenile and adult forms occur. Women are more commonly affected than men with dermatomyositis. Dermatomyositis is diagnosed based on rashes on the skin, progressive muscle weakness, elevated serum muscle enzymes, and abnormal electromyogram findings. "neck-line Vshaped" describes the characteristic skin lesions of dermatomyositis. 4 Cutaneous symptoms include photosensitivity and pruritis. Muscle symptoms can be mild to severe weakness, muscle cramps, and fatigue. Dyspnea, dysphagia, and weight loss are commonly found in progressive disease or malignancy. However, this summarised review article is covering all facts about the disease which is very much relevant for researchers and physicians. This article is also very much clinically significant. ${ }^{(6)(14)}$

Acknowledgement: None

\section{Conflict of Interest: None}

\section{Source of Funding: None}

\section{REFERENCES}

1. Ruhlman D, Otten C, Colella $\mathrm{C}$ et al. Dermatomyositis . Journal for Nurse Practitioners, 2014;,10(9): 674-681.
2. D. Ravi, S.Prabhu et al. Dermatomyositis: A dermatological perspective .Clinical Dermatology Review. 2019 ;3(1) :18

3. S. Pokhrel, B. Pardhe, N. Giri, et al. Classical dermatomyositis: A case report. Clinical, Cosmetic and Investigational Dermatology .2020; 13: 123-126.

4. Madeline E. De Wane, B.A., Reid Waldman, M.D., Jun Lu, M.D. Dermatomyositis Part I: Clinical Features and Pathogenesis. Journal of the American Academy of Dermatology.2020; 82(2) :267281.

5. Reid Waldman, M.D., Madeline E. De Wane, B.A., Jun Lu, M.D .Dermatomyositis Part 2: Diagnosis and Treatment, Journal of the American Academy of Dermatology. 2020;82(2): 283-296.

6. Davidson, Principle and practice of medicine, edited by Haslett Chilvers Edwin R. et al. Nineteenth Edition, 2002, 866.

7. Koler RA, Montemarano A. Dermatomyositis. Am Fam Physician. 2001; 64(9):1565-1572.

8. Marvi U, Chung L, Fiorentino DF. Clinical presentation and evaluation of dermatomyositis. Indian J Dermatol. 2012; 57(5):375-381.

9. Habif TP. Connective tissue diseases. In: Clinical Dermatology. 5th ed. Philadelphia, PA: Elsevier Health Sciences; 2009:671709.

10. Reddy BY, Hantash BM. Cutaneous connective tissue diseases: epidemiology, diagnosis, and treatment. Open Dermatol J. 2009;3(1):22-31.

11. Sunkureddi P, Nguyen-Oghalai TU, Jarvis JL, Karnath BM. Clinical signs of dermatomyositis. Hosp Physician. 2005;41(3):41-44.

12. Femia AN, Vleugels RA, Callen JP. Cutaneous dermatomyositis: an updated review of treatment options and internal associations. Am J Clin Dermatol. 2013;14(4):291-313.

13. Lam C, Vleugels RA. Management of cutaneous dermatomyositis. Dermatol Ther . 2012;25(2):112-134.

14. Sontheimer RD. Dermatomyositis: an overview of recent progress with emphasis on dermatologic aspects. Dermatol Clin. 2002;20(3):387-408.

15. Ballanti E, Perricone C, Greco E, et al. Complement and autoimmunity. Immunol Res. 2013;56(2-3):477-491. 
R. Ghosh et.al. Summarized review on dermatomyositis: demonstrating modes of diagnosis \& current therapeutic managements.

16. Joshi R. Interface dermatitis. Indian $\mathrm{J}$ Dermatol Venereol Leprol. 2013;79(3): 349-359.

17. Zaba LC, Fiorentino DF. Skin disease in dermatomyositis. Curr Opin Rheumatol. 2012;24(6):597-601.

18. Goreshi R, Chock M, Foering $\mathrm{K}$, et al. Quality of life in dermatomyositis. J Am Acad Dermatol. 2011;65(6):1107-1116.

19. Sigurgeirsson B, Lindelöf B, Edhag O, Allander E. Risk of cancer in patients with dermatomyositis or polymyositis. A population-based study. $\mathrm{N}$ Engl $\mathrm{J}$ Med. 1992;326(6):363-367.

20. Lodish MB. Dermatology: what's your dx? Contemp Pediatr. 2006;23(9):24-25.
21. Mahil S, Marks D, Mccormack M, Rahman A. Dermatomyositis. $\mathrm{Br} \mathrm{J}$ Hosp Med (Lond). 2012;73(2):C18-C22.

22. Chen Y, Wu C, Shen J. Predicting factors of malignancy in dermatomyositis and polymyositis: a case-control study. $\mathrm{Br} \mathrm{J}$ Dermatol. 2001;144(4):825-831.

How to cite this article: R. Ghosh, D. Ghosh. Summarized review on dermatomyositis: demonstrating modes of diagnosis \& current therapeutic managements. International Journal of Research and Review. 2021; 8(6): 304-313. DOI: https://doi.org/10.52403/ijrr.20210638 